\title{
Multi-Model Adaptive Predictive Control System for Automated Regulation of Mean Blood Pressure
}

\author{
https://doi.org/10.3991/ijoe.v15i11.10912 \\ Humberto A. Silva ${ }^{(凶)}$ \\ Federal Institute of Rio Grande do Norte, Natal/RN, Brazil \\ humberto.araujo@ifrn.edu.br \\ Celina P. Leão, Eurico A. Seabra \\ University of Minho, Guimarães, Portugal.
}

\begin{abstract}
After cardiac surgery operation, severe complications may occur in patients due to hypertension. To decrease the chances of complication it is necessary to reduce elevated mean arterial pressure (MAP) as soon as possible. Continuous infusion of vasodilator drugs, such as sodium nitroprusside (Nipride), it is used to reduce MAP quickly in most patients. For maintaining the desired blood pressure, a constant monitoring of arterial blood pressure is required and a frequently adjust on drug infusion rate. The manual control of arterial blood pressure by clinical professionals it is very demanding and time consuming, usually leading to a poor control quality of the hypertension. The objective of the study is to develop an automated control procedure of mean arterial pressure (MAP), during acute hypotension, for any patient, without changing the controller. So, a multi-model adaptive predictive methodology was developed and, for each model, a Predictive Controller can be a priori designed (MMSPGPC). In this paper, a sensitivity analysis was performed and the simulation results showed the importance of weighting factor $(\varphi)$, which controls the initial drug infusion rate, to prevent hypotension and thus preserve patient's health. Simulation results, for 51 different patients, showed that the MMSPGPC provides a fast control with mean settling time of 04:46 min, undershoots less than $10 \mathrm{mmHg}$ and steady-state error less than $\pm 5 \%$ from the MAP setpoint.
\end{abstract}

Keywords-Blood Pressure Control, Multi-Model, Predictive Control, Sodium Nitroprusside

\section{Introduction}

Postsurgical complications of hypertension can occur, or to be aggravated, in cardiac patients. To decrease the probability of complications it is necessary to reduce, at the earliest possible stage, the high blood pressure. A way to reach this objective is to use a continuous infusion of vasodilator drugs, such as sodium nitroprusside (SNP) that can quickly lower the blood pressure in most patients, bearing in mind that an overdose of nitride could cause toxic side effects. 
Drug Delivery Systems are the devices that are used to infuse the drug into the human body at a particular rate for a given time period. These systems are widely used in cardiovascular surgical treatments and Intensive Care Units (ICU). The drugs that are used during treatments are mainly used to control the blood pressure. Control of such drugs during surgeries and in ICU are very tedious since manual control are done by anesthetists which is not accurate and takes time [1]. Manual control may be time consuming, and of poor quality. Due to disturbances that perturb pressure, the changing conditions of patient, and the wide range of response characteristics determining the right drug infusion may be difficult. This process causes lots of fatigue to the caregivers and increases chances for errors or missing critical events. These situations are commonplace in ICU setups. In recent times this situation is being avoided by the use of automated drug infusion systems based on therapeutic models. These can improve the drug delivery and can ease the burden of continual monitoring of infusion, volume adjustments, data logging, and documentation [2]. Many investigators have reported on the use of automated systems for blood pressure control $[3,4,5,6]$ and multiple drug infusion systems to regulate hemodynamics such as cardiac output and mean arterial blood pressure (MAP) [7], [8].

The objective of this paper is to develop an adaptive method control for a blood pressure management for any patient without changing the controller. Blood pressure control of a patient under the influence of the vasodilator SNP is modelled through an uncertain model [9], [10]. A multi-model approach is used in order to control the blood pressure under the influence of this drug. Multi-model approaches are commonly applied to control non-linear systems that operates in long ranges [11], [12]. The basic idea of multi-model approach consists in decompose the system's operating range into a number of operating regimes that completely cover the chosen trajectory [12]. There are, basically, two approaches for multi-model. The first one consists of designing a set of suitable controllers (one for each operating regime) and to calculate weighting factors to them as showed by the study by [12]. Moreover, an sensitivity analysis of the parameter $(\varphi)$ which regulates the initial drug infusion rate was performed.

\section{Problem Formulation}

The pressure exerted by circulating blood upon the blood vessels is termed as Blood Pressure (BP). It usually refers to the arterial pressure of the systemic circulation. During each heartbeat, blood pressure varies between a maximum and a minimum pressure [13]. The pressure exerted due to pumping of heart causes systolic blood pressure (SBP) and the pressure exerted by blood vessels causes' diastolic blood pressure (DBP). The blood pressure in circulation is principally due to heart rate. A person's blood pressure is usually expressed in terms of the systolic pressure over diastolic pressure and is measured in millimeters of mercury $(\mathrm{mmHg})$, for example $120 / 80$. Blood pressure that is pathologically low is called hypotension and which is pathologically high is hypertension. In most cases, we measure the mean arterial pressure (MAP) by using equation (1) [13]. 


$$
M A P=[S B P+(D B P \times 2)] / 3
$$

After cardiac operation, severe complications may occur in patients due to hypertension. It may damage heart cells, causes excessive bleeding, bursting of veins in brains etc. To decrease the chances of complication, the MAP must be maintained at a desired level. This is achieved by intravenous infusion of suitable vasodilator drugs such as sodium nitroprusside (SNP), Nitroglycerin, etc., which are commonly used for the treatment of hypertensive cardiac patients. The infusion of these drugs will generate Nitric Oxide (NO) and there by reduces the MAP. It causes widening of blood vessels and has less side effects [14].

The American College of Cardiology (ACC)/American Heart Association (AHA) hypertension guideline published in November 2017 introduced new blood-pressure categories lowering the threshold for the diagnosis of hypertension. Where, Hypertension is defined by a systolic blood pressure greater than $130 \mathrm{mmHg}$ or a diastolic pressure greater than $80 \mathrm{mmHg}$. Table 1 shows the blood pressure classification established by [15]. The categories of blood pressure from normal to stage 2 hypertension.

Table 1. Blood pressure classification (from [15])

\begin{tabular}{|l|c|l|c|}
\hline \multicolumn{1}{|c|}{ BP Category } & SBP $(\mathbf{m m H g})$ & & DBP $(\mathbf{m m H g})$ \\
\hline Normal & $<120$ & and & $<80$ \\
\hline Elevated BP & $120-129$ & and & $<80$ \\
\hline Stage 1 hypertension & $130-139$ & or & $80-89$ \\
\hline Stage 2 hypertension & $\geq 140$ & or & $\geq 90$ \\
\hline
\end{tabular}

\subsection{Blood pressure control systems}

The objective of the control system is to regulate the MAP at any setpoint and maintain the setpoint determined in the presence of undesired disturbances and changing conditions of patient. Since it is desirable to develop a system that will be used in clinical applications, it is essential to establish realistic design specifications. In general, the control system must be able to control MAP as quickly as possible, while meeting control specifications, increasing system security and preserving patient health.

The closed loop control system should respond quickly and smoothly to changes in the MAP setpoint (performed by the health care professional) without a excessive overshoot. The closed loop system shall minimize the effects of undesired disturbances, such as measurement errors, resulting from calibration errors and stochastic background noise. Since it is desired to use the same control system for many different patients and it is not possible to have a separate model for each patient (for practical reasons), it should have a closed-loop system that is insensitive to changing conditions of patient (it must meet the specifications for any patient).

Based on clinical experiences [9], it is possible to determine the control specifications as follows:

- Settling time less than 15 minutes;

- Overshoot less than $15 \%$; 
- Steady-state error less than $\pm 5 \%$ from the MAP setpoint;

- Minimum sensitivity to changing conditions of patient.

Figure 1 show the main elements of the system with controller, infusion pump, sensor and patient. The system input $R(s)$ is the MAP setpoint, and the output $Y(s)$ is the actual change in pressure. The difference between the desired change and the measured change in blood pressure forms a signal used by the controller to determine set values for the infusion pump, which is responsible for infusing the drug into the patient.

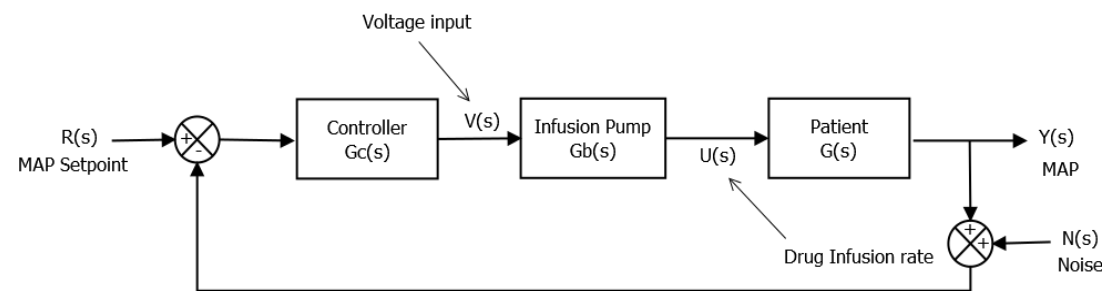

Fig. 1. Blood Pressure Control System

\subsection{Infusion pump model}

The infusion pump model depends directly on the mechanical design. A simple infusion pump is allowed in which the infusion rate variation at the output is equal to the input voltage adjustment at the pump:

$$
\dot{u}(t)=v(t)
$$

Therefore, the transfer function of the pump is given by:

$$
G_{b}(s)=\frac{U(s)}{V(s)}=\frac{1}{s}
$$

This is equivalent to saying that from an input/output perspective, the infusion pump has the impulse response $h(t)=1$, for $t \geq 0$.

\subsection{Patient modeling}

A model for the mean arterial pressure (MAP) of a patient under the influence of sodium nitroprusside (Nipride) can be represented, as in [9], by:

$$
M A P(t)=P_{0}(t)-\Delta P(t)+v(t)
$$

Where $P_{0}$ is the initial blood pressure, also called a background pressure, $P(t)$ is the pressure differential due to infusion of Nipride, and $v(t)$ is a stochastic background noise. In this paper it is assumed that $P_{0}$ is constant. A continuous-time deterministic model describing the relationship between the change in the blood pressure, $\Delta P(s)$, and drug infusion rate, $I(s),[9]$ is as follows: 


$$
\Delta P(s)=\frac{K e^{-T i^{s}}\left(1+\alpha e^{-T_{c} s}\right)}{1+\tau s} I(s)
$$

Where $K$ is drug sensitivity, $\alpha$ is the recirculation constant, $T_{i}$ is the initial transport delay, $T_{c}$ is the recirculation time delay, and $\tau$ is a time constant. The corresponding discrete-time deterministic model for this process can be given as follows:

$$
\Delta P(t)=\frac{q^{-d}\left(b_{0}+b_{m} q^{-m}\right)}{1-a_{1} q^{-1}} I(t) ; b_{0}>0
$$

Where $\mathrm{q}^{-1}$ denotes a unit delay operator, parameters $\mathrm{b}_{\mathrm{o}}, \mathrm{b}_{\mathrm{m}}, \mathrm{a}_{1}, \mathrm{~d}$, and $\mathrm{m}$ are obtained from the sampled version of the continuous-time model given in (5). A range of typical values for the parameters of the model (5) for different patients is given by [9] and are found in Table 2.

Table 2. Range of values for parameters of the continuous patient model [9]

\begin{tabular}{|c|c|c|c|}
\hline Parameter & Minimum & Maximum & Nominal \\
\hline$K$ & 0.25 & 9 & 1 \\
\hline$\alpha$ & 0 & 0.4 & 0.1 \\
\hline$\tau(\mathrm{s})$ & 30 & 60 & 40 \\
\hline$T_{i}(\mathrm{~s})$ & 20 & 60 & 40 \\
\hline$T_{c}(\mathrm{~s})$ & 30 & 75 & 45 \\
\hline
\end{tabular}

For a given patient, time delays are unknown, but are assumed to be constant over a long period of time whereas the parameters $K, \alpha$ and $\tau$ change during the infusion procedure. In this work, it is assumed that the parameters change in an exponential manner and modeled as follows [5]:

$$
\operatorname{par}(k)=\operatorname{par}(0)\left(2-e^{-k / \gamma}\right)
$$

For increase and decrease in the parameter value, respectively, where par(t) represents the parameter of the continuous-time model and $\gamma$ is the change time constant. Thus, the controller when tuned for a particular patient, should be able to handle timevarying parameters and initially unknown time delays.

\section{Smith Predictor Based Generalized Predictive Control}

The Smith Predictor (SP), the first control system proposed in the literature that introduces a delay compensator [16], improves the performance of a system with a delay compared to other techniques, such as PID, especially when the delay is dominant (greater than twice the dominant time constant of the system).

Using a SP instead of an optimal predictor in generalized predictive controllers (GPC) for stable plants and in real applications have advantages [17], [18], [19], namely in the case of time-delay systems. Setting the GPC algorithm, it is possible to improve the robustness of the closed-loop system while maintaining the nominal performance. A Smith Predictor Based Generalized Predictive Control (SPGPC) uses a Smith predictor structure to compute the predictions of the output of the plant and to 
calculate a sequence of future control signals in order to minimize a multistage cost function defined over a control horizon, as described in [17].

\section{Multiple Model SPGPC Method}

The high variability between patients as well as the variability within the same patient on different days and even within the same patient during a given experimental run, is the greatest control challenge for this system. This became evident in the first few canine experiments using a fixed prediction model derived from a nonlinear model [20] and step responses tests performed on the canine. So, a different approach based on using multiple models was considered. In Rao et al. [20] a considerable amount of research in a Multiple Model Adaptive Control (MMAC) framework using a bank of several model controller pairs was performed.

The Multiple Model SPGPC (MMSPGPC) procedure, shown in Figure 2, is a control strategy that can identify and then adapt to changing characteristics. The MMSPGPC uses a finite range of models (assumed to be reasonable representations of possible patients) from which to identify the patient. Single-model adaptive control schemes using some sort of recursive parameter estimation have, in essence, an infinite range of models from which to identify the patient. MMSPGPC has an advantage over single-model schemes, because it limits the identification to one of a finite set of possible models, all of which are realistic [4]. The MMSPGPC is based upon the assumption that the patient can be represented by a finite number of models and, for each model a predictive controller can be priori designed. An adaptive mechanism is then needed to decide which controller should be dominant for a given patient. One procedure for solving this problem is to consider a weighted sum of all the controller outputs, where the weighting factors are determined by the relative residuals between the patient response and the model responses [21].

In Figure 2, the system error, e, is expressed as:

$$
e(k)=p(k)-p_{c}
$$

Where $k$ is the sampling time and $p_{c}$, is the commanded or setpoint pressure level.

\subsection{Controller bank design}

The controller bank contains a set of Smith Predictor Based Generalized Predictive Controllers where for each model, present in the model bank, a corresponding SPGPC has been tuned. The SPGPC uses a smith predictor structure to compute the predictions of the system output and to calculate a sequence of future control signals in order to minimize a multistage cost function defined over a control horizon, as follows [21]:

$$
J\left(N_{1}, N_{2}\right)=\sum_{j=N_{1}}^{N_{2}} \delta(j)[\hat{y}(t+j \mid t)-w(t+j)]^{2}+\sum_{i=1}^{N_{2}-d} \lambda(i)[\Delta u(t+i-1)]^{2}(9)
$$




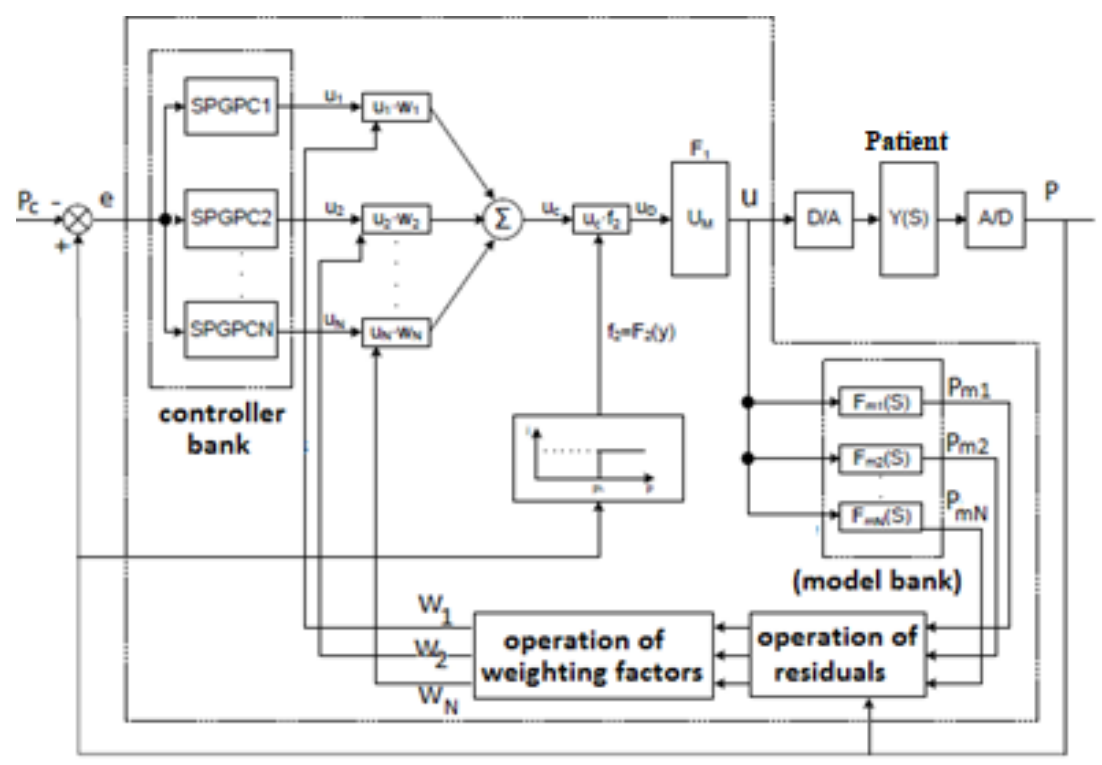

Fig. 2. Multiple Model SPGPC Schema (Adapted from [21])

Where $N_{1}$ and $N_{2}$ are the minimum and maximum costing horizons, respectively, $\mathrm{d}$ is the delay of the process model, $\delta(\mathrm{j})$ and $\lambda(\mathrm{j})$ are weighting sequences, $w(\mathrm{t}+\mathrm{j})$ is a future set-point or reference sequence, $\Delta \mathrm{u}(\mathrm{t})$ is the incremental control action $\Delta \mathrm{u}(\mathrm{t})=$ $\mathrm{u}(\mathrm{t})-\mathrm{u}(\mathrm{t}-1)$ and $(\mathrm{t}+\mathrm{j} \mid \mathrm{t})$ is the $\mathrm{j}$-step ahead prediction of the system output on data up to time $t$. The performance of the predictive controller depends on the proper tuning of the prediction horizon $N_{2}$, and the weighting factor $\lambda$. Using a non-automatic tuning method, an initial choice of the parameters was performed and then modified until the project specifications were met. This final adjustment is done by trial and error, based on the fact that the increase in $\lambda$ decreases the magnitude (effort) of the control signal, making the response slower and smoother. However, the increase in $\lambda$ provides greater robustness. The simultaneous increase in the prediction and control horizons contributes to improve controller stability, undershoot and settling time. According to [22] the value of the prediction horizon must be equal:

$$
N_{2}=d_{\max }+\frac{T_{S} / T}{3.5}
$$

Where $T$ is the sampling time, $d_{\max }$ is the number of samples contained in the maximum estimated dead time and $T_{\mathrm{s}}$ is the settling time. Thus, after calculating the prediction horizon, the $\lambda$ were tuned by trial and error.

\subsection{Model bank design}

The model bank consists of a number of models with constant parameters characterizing the individual patient subspace [23]. Since these models should have the same structure as the patient, the following discrete model will describe them: 


$$
\Delta P_{m j}(k)=\frac{q^{-d}\left(b_{0 j}+b_{m j} q^{-m}\right)}{1-a_{1 j} q^{-1}} u(k) ; b_{0 j}>0(j=1, \ldots, N)
$$

Where $N$ is the number of models and the output pressure from model $j$ is:

$$
P_{m j}(k)=\Delta P_{m j}(k)+P_{0} \quad(\mathrm{j}=1, \ldots, \mathrm{N})
$$

Where $\Delta P_{m j}(k)$ is the change in the $j t h$ model output, $u(k)$ is the model input, $P_{0}$ is the initial value of each model's output and equals the initial system output.

The relative residual $R_{j}^{2}(k)$ will be defined as the normalized squared error between patient and model, i.e., as follows:

$$
R_{j}^{2}(k)=\left\{\left[P_{m j}(k)-P(k)\right] /\left(P_{0}-P_{c}\right)\right\}^{2} \quad(\mathrm{j}=1, \ldots, \mathrm{N})
$$

At each sample time $k$, the model that has the smallest residual is defined as the matching model, which is used to represent the patient $t$ characteristics.

To determine the number of models needed and the range of drug sensitivity that each model could cover, a SPGPC was set up. Then to find the maximum drug sensitivity that the model could control, drug sensitivity of the patient was increased until a 10 percent overshoot occurred or a settling time more than 400 seconds. This was the maximum drug sensitivity allowed for the model. Figure 3 shows the flowchart describing the algorithm to determine the range of drug sensitivity that each model could cover. 


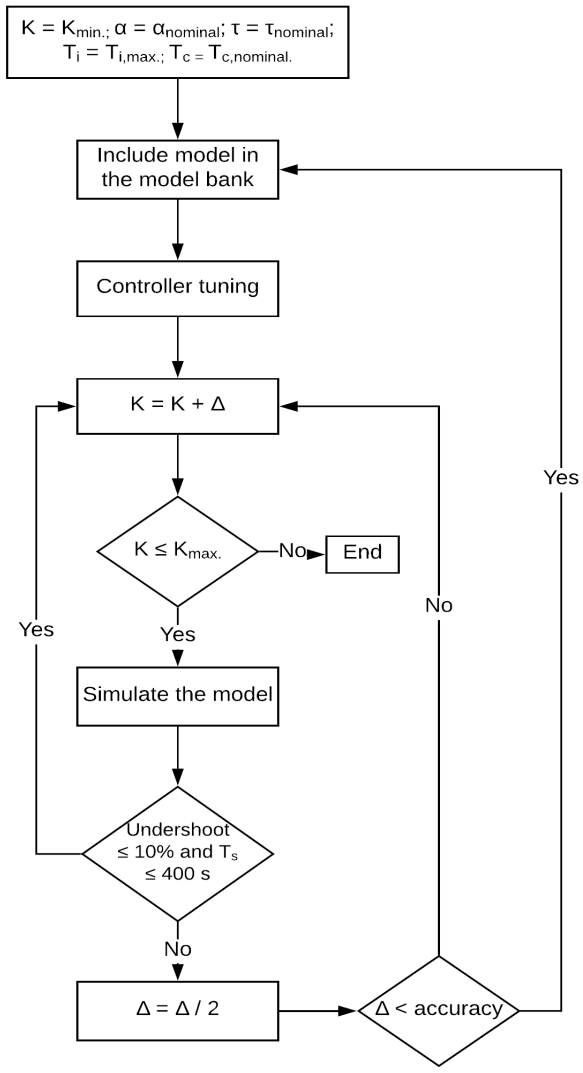

$K_{\min .}$ the minimum drug sensitivity;

$\alpha_{\text {nominal }}, \tau_{\text {nominal }}$ and $\mathrm{T}_{\mathrm{c}, \text { nominal }}$ the nominal values of the parameters;

$\mathrm{T}_{\mathrm{i}, \mathrm{max}}$, the maximum time delay;

$\mathrm{T}_{\mathrm{s}}$ the settling time;

$\Delta$ the increase parameter (initially high and decreases until reaching the wanted accuracy value)

Fig. 3. Algorithm to determine the range of drug sensi

\subsection{Control algorithm}

To reach desirable system performance and to guarantee patient safety, the control algorithm should converge quickly to the optimal values and should react to time varying patient characteristics, as well as ensure a reasonable rate of blood pressure change. Thus, the control, $u_{c}(k)$, was computed as a weighted sum of controller bank signals, and represented by the following equation:

$$
u_{c}(k)=\sum_{j=1}^{N} W_{j}(k) u_{j}(k)
$$

Where $N$ is the number of models, $u_{j}(k)$ are the individual controller outputs and $W_{j}(k)$ are the weighting factors. The weights were selected as follows: 
- Recursive update

$$
W_{j}^{\prime}(k)=\frac{\exp \left[-R_{j}^{2} / 2 V\right] W_{j}(k-1)}{\sum_{i=1}^{N} \exp \left[-R_{j}^{2} / 2 V\right] W_{j}(k-1)}
$$

- Bounding away from zero

$$
W_{j}(k)=\left\{\begin{array}{cc}
W_{j}^{\prime}(k) & W_{j}^{\prime}(k)>\delta \\
\delta & W_{j}^{\prime}(k) \leq \delta
\end{array}\right.
$$

- Normalization

$$
W_{j}(k)=\frac{\left[W_{j}(k)\right]^{2}}{\sum_{i=1}^{N}\left[W_{j}(k)\right]^{2}}
$$

Where $R_{j}(k)$ are the residuals and defined in (13), $V$ is a parameter controlling the convergence rate of $W_{j}^{\prime}(k)$ with $R_{j}(k)$ and $\delta$ is a threshold to limit the importance of past information. Equations (14) and (15) express the basic relationship between the control, the weighting factors, and the relative residuals. Equation (16) is used to delimit the importance of past information enabling the adaptive mechanism quickly react to the new information about the patient characteristics. Equation (17) is used to normalize the weighting factors so that their square sum is equal to unity.

From (15), it is observed that a large value of $\delta$ will improve the sensitivity of the algorithm to the new patient information. The parameter $V$ in (15) plays an important role in controlling the convergence rate of $W_{j}(k)$. Since the drug sensitivity may be located in any position in the patient parameter space, the values for $\mathrm{W}_{\mathrm{j}}(0)$ were assumed to be uniform and calculated by:

$$
W_{j}(0)=W_{j}^{\prime}(0)=\frac{1}{N}(j=1, \ldots, N)
$$

\subsection{Safety functions}

For patient safety, two nonlinear units, $F_{1}$ and $F_{2}$, are built into the system. The nonlinear unit limiting infusion rate is given by

$$
u=F_{1}\left(u_{D}\right)= \begin{cases}0, & \text { if } u_{D}<0 ; \\ u_{D}, & \text { if } u_{D} \leq U_{M} ; \\ U_{M}, & \text { if } u_{D}>U_{M}\end{cases}
$$

Where $U_{M}$ is the allowed maximum infusion rate. For patient safety, the infusion rate should be reduced under hypotension, i.e., when there is a drop-in excess of 20 $\mathrm{mmHg}$ from the set point. Thus, other nonlinear unit is used to turn off the infusion if and when hypotension occurs. Its expression is given by:

$$
F_{2}(p(k))= \begin{cases}1, & \text { for } p(k) \geq p_{L} \\ 0, & \text { for } p(k)<p_{L}\end{cases}
$$


where $\mathrm{p}_{\mathrm{L}}$ is defined as:

$$
p_{L}=p_{c}-20
$$

\subsection{Regulating the initial infusion rate}

Since the control variable $u_{c}(k)$ calculated by (14) will be in error before the convergence of $W_{j}(k)$, and this error can cause a large undershot for patients with high drug sensitivity, the algorithm consisting of (14) to (17) was modified as follows:

$$
u_{D}(k)=\left\{\begin{array}{c}
\varphi u_{c}(k), \text { for } k \leq d_{m} \\
{\left[\varphi+\varphi_{1}\left(k-d_{m}\right)\right] u_{c}(k), \text { for } d_{m}<k<\left(d_{m}+D\right)} \\
u_{c}(k), \text { for } k \geq\left(d_{m}+D\right)
\end{array}\right.
$$

Where $\varphi$ and $\varphi_{1}$ are positive coefficients and less than $1, D=\operatorname{INTEGER}\left[\frac{1-\varphi}{\varphi_{1}}\right]$, and $d_{m}=T_{i}(m a ́ x) /$.$T with T$ the sampling time. $\varphi$ is used to regulate the initial infusion rate so as to prevent large undershoot. The value of $\varphi$ should place the initial control variable near the steady-state control input required by the patient with the highest expected drug sensitivity. $\varphi_{1}$ is then set so that the increment of the initial infusion rate ensures smooth reduction of pressure. Note that because of this conservative initial control policy, a large error could persist and subsequently cause an undershoot of the system output. To eliminate this behavior, the $j t h$ model error $e_{m j}(k)$ was decreased during the initial control period as follows:

$$
e_{m j}(k)=\beta_{m j}(k) \times\left(P_{m j}(k)-p_{c}\right) ; \text { for }(\mathrm{j}=1, \ldots, \mathrm{N})
$$

where $\beta_{m j}$ are modifying coefficients defined as

$$
\left\{\begin{array}{l}
\beta_{m j}(k) \leq 1 \text { para } k \leq d_{m}+D \\
\beta_{m j}(k)=1 \text { para } k>d_{m}+D
\end{array}\right.
$$

\section{$5 \quad$ Results and Discussion}

\subsection{Computer simulation}

Computer simulations were used to evaluate the response of the system design (figure 2 a representative patient parameter envelope. It was studied the response to step command in the presence of system background noise, the adaptation of the algorithm to time-varying patient parameter.

Table 3 shows the model bank and controller bank, which were obtained from the algorithm in figure 3 where the 8 models used in the model bank and their respective controllers were obtained. 
Table 3. Parameters of Controllers and Models

\begin{tabular}{|c|c|c|c|c|c|c|c|}
\hline $\mathrm{N}^{0}$ & \multicolumn{2}{|c|}{ Controller } & \multicolumn{5}{|c|}{ Model } \\
\hline & $\lambda$ & $\mathrm{N}_{2}$ & $\boldsymbol{K}$ & $T_{i}$ & $\boldsymbol{T}_{c}$ & $\bar{\tau}$ & $\alpha$ \\
\hline 1 & 3 & \multirow{8}{*}{10} & 0.25 & \multirow{8}{*}{60} & \multirow{8}{*}{45} & \multirow{8}{*}{40} & \multirow{8}{*}{0.4} \\
\hline 2 & 6 & & 0.37 & & & & \\
\hline 3 & 8 & & 0.53 & & & & \\
\hline 4 & 18 & & 0.87 & & & & \\
\hline 5 & 30 & & 1.52 & & & & \\
\hline 6 & 80 & & 2.26 & & & & \\
\hline 7 & 150 & & 3.51 & & & & \\
\hline 8 & 250 & & 5.66 & & & & \\
\hline
\end{tabular}

The coefficient $V$ in (15) was determined by computer simulation as described in [12]. Thus, $V$ was chosen to be 0.05 . The initial weighting factors were assumed to be uniform and calculated by (18):

$$
W_{j}(0)=\frac{1}{8}
$$

The parameter that regulate the initial infusion rate $(\varphi$ in (22)), was determined by computer simulation (as described in subsection 5.2 Parametric sensitivity analysis). The results obtained by sensitivity analysis showed that the undershoot specification can be satisfied when $\varphi=\varphi_{1}=0.2$.

The modification of $\beta_{m j}$ is needed only for the control of lower drug sensitivity, so that some components of $\beta_{m j}$, such as $\beta_{m 5}, \ldots, \beta_{m 8}$, corresponding to the controller outputs for high drug sensitivity, can directly be set to unity. The other components, $\beta_{m l}, \ldots, \beta_{m 4}$, were determined by trial and error. Their values are listed in Table 4.

Table 4. Parameters of control algorithm

\begin{tabular}{|c|c|c|c|c|c|c|c|}
\hline $\mathbf{V}$ & $\boldsymbol{\delta}$ & $\boldsymbol{W}_{\boldsymbol{j}}(\mathbf{0})$ & $\boldsymbol{\varphi}$ & $\boldsymbol{\varphi}_{\boldsymbol{1}}$ & $\boldsymbol{d}_{\boldsymbol{m}}$ & $\mathbf{D}$ & \\
\hline 0.05 & 0.01 & $1 / 8$ & 0.2 & 0.2 & 6 & 4 & \\
\hline $\boldsymbol{\beta}_{\boldsymbol{m} \mathbf{1}}$ & $\boldsymbol{\beta}_{\boldsymbol{m} \mathbf{2}}$ & $\boldsymbol{\beta}_{\boldsymbol{m} \mathbf{3}}$ & $\boldsymbol{\beta}_{\boldsymbol{m} \mathbf{4}}$ & $\boldsymbol{\beta}_{\boldsymbol{m} \mathbf{5}}$ & $\boldsymbol{\beta}_{\boldsymbol{m} \mathbf{6}}$ & $\boldsymbol{\beta}_{\boldsymbol{m} \mathbf{7}}$ & $\boldsymbol{\beta}_{\boldsymbol{m} \mathbf{8}}$ \\
\hline 0.75 & 0.85 & 0.85 & 0.85 & 1 & 1 & 1 & 1 \\
\hline
\end{tabular}

The regime blood pressure considered was $150 \mathrm{mmHg}$ and the multi-model controller deviation reference of $-50 \mathrm{mmHg}$. The system background noise $v(t)$ was simulated as a white Gaussian noise sequence with standard deviation of $4 \mathrm{mmHg}$. The simulations were performed with the patient parameters: $\mathrm{T}_{\mathrm{c}}=50 \mathrm{~s}, \tau,=45 \mathrm{~s}, \alpha=0.4$, as well as for the different values of drug sensitivity $(K)$ and patient delay time $\left(\mathrm{T}_{\mathrm{i}}\right)$. The blood pressure $(\mathrm{mmHg})$, the deviation in infusion rate $(\mathrm{ml} / \mathrm{h})$ and the weighting factors convergence of several patients with the parameters: $T_{c}=50 \mathrm{~s}, \tau,=45 \mathrm{~s}, \alpha=$ 0.4 , as well as for different drug sensitivity $(0.25 \leq K \leq 9)$ and patient delay time $\left(T_{i}=\right.$ 20,40 or $60 \mathrm{~s}$ ) obtained are shown in figures 4 to 7 . In all cases the drug sensitivity and delay time were considered exponentially time-varying during the procedure by Equation (7).

Considering the data obtained by simulations (figures 4 to 7 ) the convergence process of the weighting factors $W_{j}(k)$, the global control effort is calculated to the closest model (less residual error), as such as the schema leads the blood pressure of the cho- 
sen patient to the given reference, making drug infusion rate and the blood pressure to change in a smooth way. These responses also show the robustness of Multi-Model SPGPC algorithm even in the presence of the system background noise and variation of patient parameters. Simulation results, for 51 different patients, showed that the MMSPGPC provides a fast control with mean settling time of 04:46 min. (06:00 min. maximum and 02:30 min. minimum), undershoots less than $10 \mathrm{mmHg}$ and steadystate error less than $\pm 5 \%$ from the MAP setpoint.
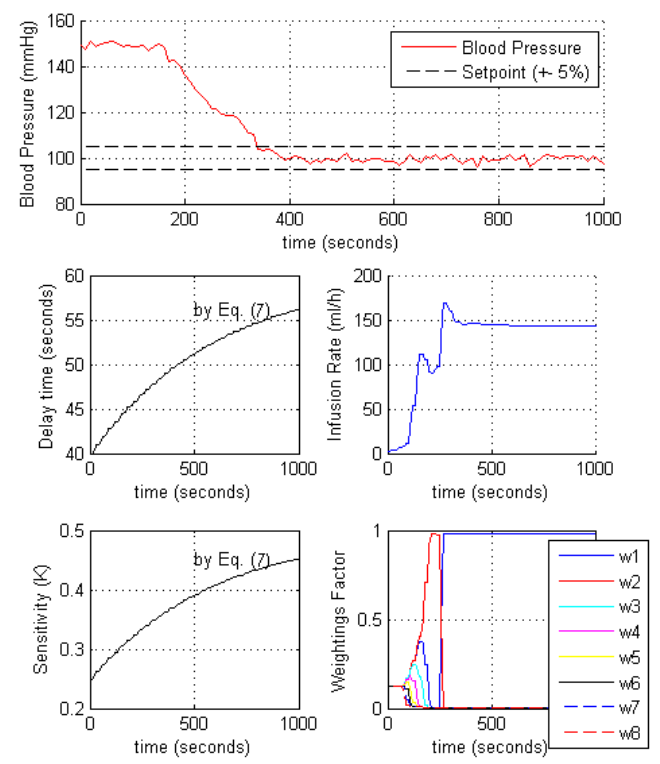

Fig. 4. Simulation results for drug sensitivity $\mathrm{K}=0.25$ and delay time $\mathrm{Ti}=40 \mathrm{~s}$

\subsection{Parametric sensitivity analysis}

The parameter $\varphi$, in equation (22), is used to regulate the initial infusion rate, in order to prevent large undershoots. The value of $\varphi$ should place the initial control variable near the steady-state control input required by the patient with the highest expected sensitivity. $\varphi_{1}$ is then set so that the increment of the initial infusion rate ensures smooth reduction of pressure. 

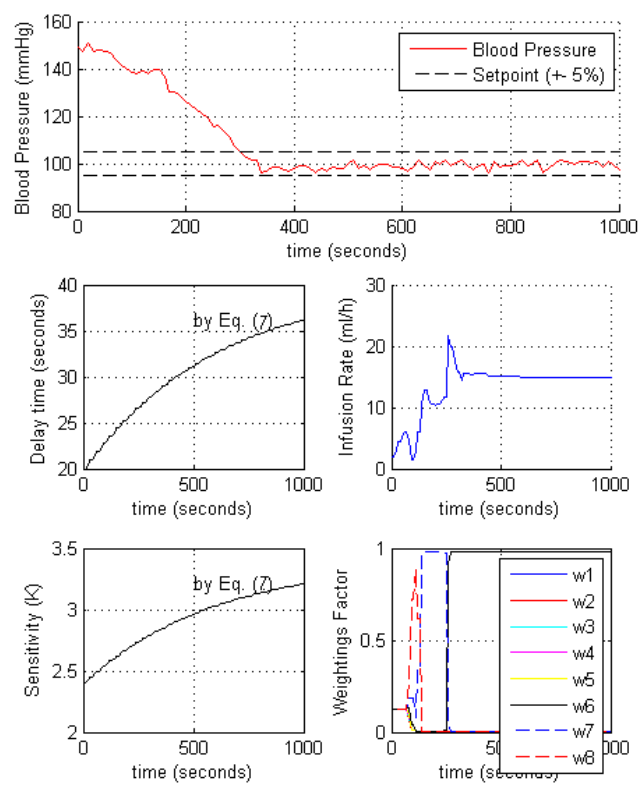

Fig. 5. Simulation results for drug sensitivity $\mathrm{K}=2.4$ and delay time $\mathrm{Ti}=20 \mathrm{~s}$
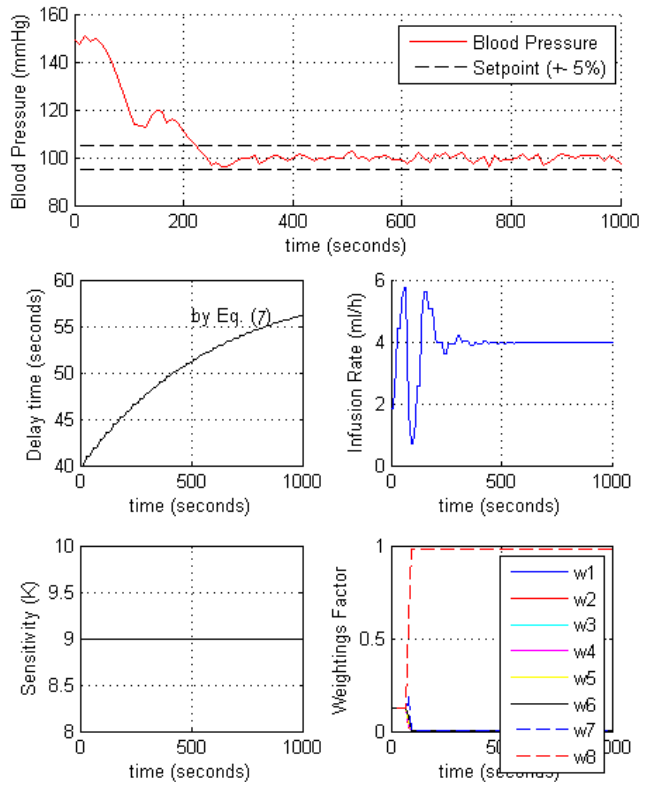

Fig. 6. Simulation results for drug sensitivity $\mathrm{K}=9.0$ and delay time $\mathrm{Ti}=40 \mathrm{~s}$ 

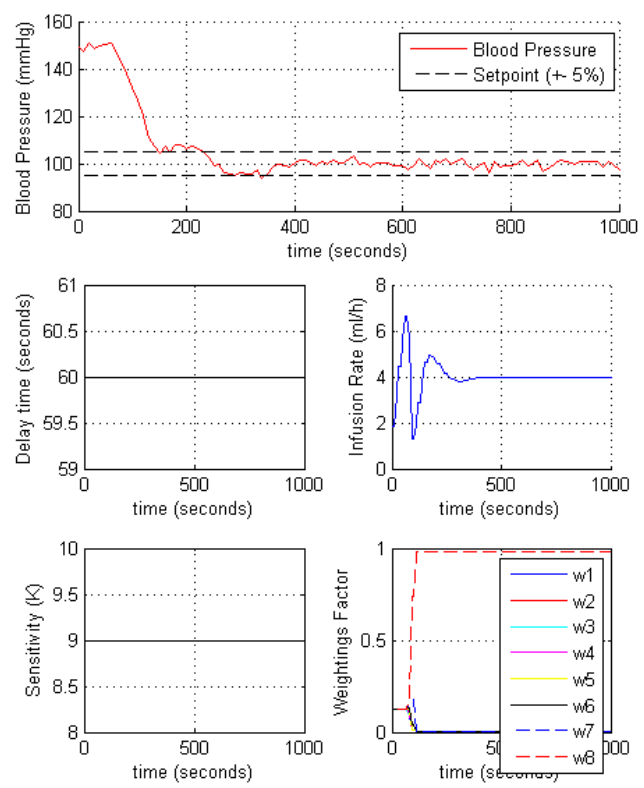

Fig. 7. Simulation results for drug sensitivity $\mathrm{K}=9.0$ and delay time $\mathrm{Ti}=60 \mathrm{~s}$

The sensitivity analysis was performed, varying $\varphi$ and verifying its influence on performance indices ( $\mathrm{T}_{\mathrm{s}}$ and undershoot). Table 5 shows the relationship between the parameter $\varphi$ and the performance indices obtained for patients with low sensitivity ( $\mathrm{k}$ $=0.25)$ and high sensitivity $(\mathrm{k}=9)$. The simulation results (see table 5) showed that without initial regulation of the drug infusion rate (i.e., $\varphi=1$ ), large undershoots ( 40.5 $\mathrm{mmHg}$ ) are observed for patients with high sensitivity. This demonstrates the need of the algorithm described in equation (22), which to reduce the undershoots for patients with high sensitivity, in order to prevent hypotension and thus preserve the patient's health.

Table 5. Relationship between the values of $\varphi$, Ts and undershoot

\begin{tabular}{|c|c|c|c|c|}
\hline & \multicolumn{2}{|c|}{$\mathbf{T}_{\mathbf{s}}$ (seconds) } & \multicolumn{2}{c|}{ Undershoot (mmHg) } \\
\hline & \multicolumn{2}{|c|}{ Drug Sensitivity } & \multicolumn{2}{c|}{ Drug Sensitivity } \\
\hline $\boldsymbol{\varphi}=\boldsymbol{\varphi}_{\mathbf{1}}$ & $\begin{array}{c}\text { Low } \\
(\boldsymbol{K}=\boldsymbol{0 . 2 5})\end{array}$ & $\begin{array}{c}\text { High } \\
(\boldsymbol{K}=\mathbf{9})\end{array}$ & $\begin{array}{c}\text { Low } \\
(\boldsymbol{K}=\mathbf{0 . 2 5})\end{array}$ & $\begin{array}{c}\text { High } \\
(\boldsymbol{K}=\boldsymbol{9})\end{array}$ \\
\hline 1 & 345 & 90 & 0 & 40.5 \\
\hline 0.5 & 330 & 105 & 0 & 21.4 \\
\hline 0.4 & 315 & 120 & 0 & 18.3 \\
\hline 0.3 & 300 & 135 & 0 & 15.2 \\
\hline 0.2 & 300 & 240 & 0 & 4.4 \\
\hline 0.1 & 315 & 270 & 6.2 & 18.1 \\
\hline 0.05 & 360 & 315 & 14.5 & 26.2 \\
\hline
\end{tabular}

The results in Table 5 shows that the undershoots are acceptable $(\leq 20 \mathrm{mmHg}$ from the setpoint) only for $0.4 \leq \varphi \leq 0.1$. 
Figure 9 show larger undershoots for patients with high sensitivity, and an exponential reduction of undershoots for lower values of $\varphi$. However, an overly conservative initial control policy $(\varphi<0.2)$, a large error could persist and subsequently cause a large undershoot.

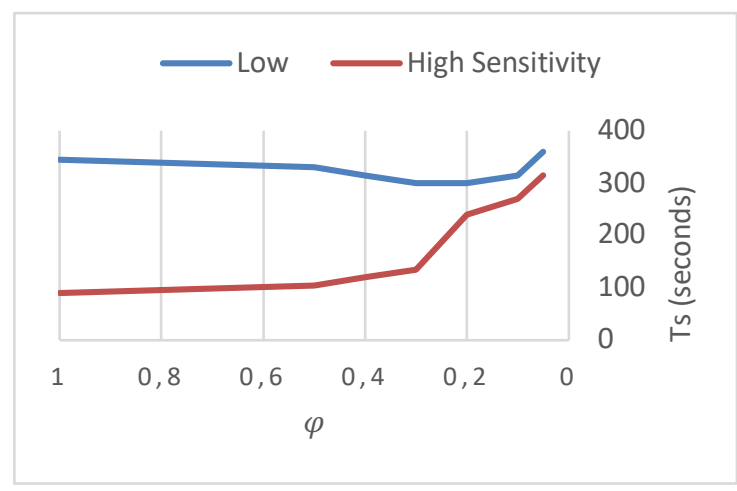

Fig. 8. Ts for the different values of $\varphi$

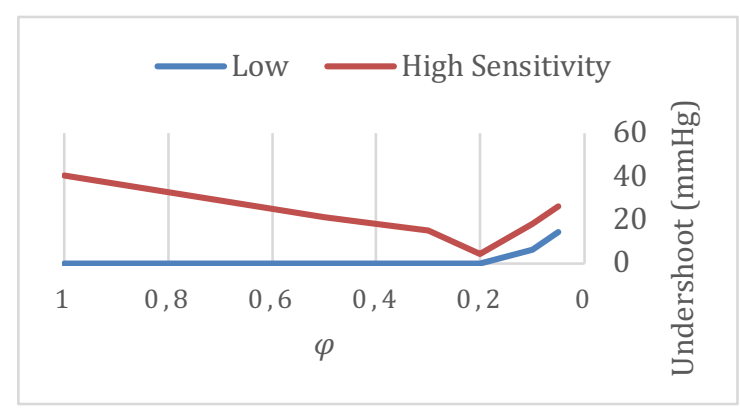

Fig. 9. Undershoot for the different values of $\varphi$

\section{Conclusion}

The simulated results show that the Multi-Model SPGPC algorithm is robust even in the presence of the system background noise and variation of patient parameters.

In an automatic blood pressure control system, large undershoots can occur, especially for patient with high drug sensitivity. When the patient has high drug sensitivity and is being controlled by a low drug sensitivity model, large overshoots will occur. In the MMSGPC method this may occur at the beginning of the control process, before the convergence of the weight factors. Therefore, it is necessary to regulate the initial infusion rate, to prevent hypotension and thus preserve the patient's health.

The developed algorithm that regulates the initial infusion rate (subsection 4.5) is very important for the MMSPGPC performance. The sensitivity analysis of the parameter $\varphi$ showed that the algorithm (described in equation (22)) was able to reduce the undershoots up to $36.1 \mathrm{mmHg}$, especially for patients with high drug sensitivity, 
where without initial reduction of the infusion rate (i.e., $\varphi=1$ ), the undershoot was $40.5 \mathrm{mmHg}$ and for $\varphi=0.2$, the undershoot was $4.4 \mathrm{mmHg}$, with a maximum settling time of 300 seconds.

In the future, robustness tests can be implemented with the submission of the system to a larger range of disturbances. Thus, the presented approach could be implemented in a microcontroller, as first approach, in tests with animals.

\section{Acknowledgement}

The authors of this article would like to thank Federal Institute of Rio Grande do Norte for support and University of Minho for structure, which to made possible the development of the research.

\section{References}

[1] G. C. Sowparnika, M. Thirumarimurugan, V. M. Sivakumar, Metaphorical analysis of Tuning rules for PI and PID Controllers in modelling an Automatic Drug Delivery System to control Mean Arterial Blood Pressure. International Conference on Advanced Computing and Communication Systems, ICACCS, Coimbatore, India, 2017. https://doi.org/ $\underline{10.1109 / \text { icaccs. } 2017.8014587}$

[2] R. Chandramouli, D. Sathyanarayana, R. Dang et al., Drug Infusions by Model Predictive Control using Computational Therapeutic Models. Asian Journal of Pharmaceutics, vol. 12, no. 3, pp. 859-862, Jul. 2018.

[3] A. A. Basha, S. Vivekanandan and P. Parthasarathy. Evolution of blood pressure control identification in lieu of post-surgery diabetic patients: a review. Health Information Science and Systems,6:17, Sep. 2018. https://doi.org/10.1007/s13755-018-0055-Z

[4] J. F. Martin, A. M. Schneider, N. T. Smith. Multiple-model adaptive control of blood pressure using sodium nitroprusside. IEEE Trans. Biomed. Eng. 34:603-611, 1987. https://doi.org/10.1109/tbme.1987.326071

[5] G. A. Pajunen, M. Steinmetz, R. Shankar, Model Reference Adaptive Control with Constraints for Postoperative Blood Pressure Management. IEEE Trans. on Biomed. Eng. vol. 37, no. 7, pp. 679-687, Jul. 1990. https://doi.org/10.1109/10.55674

[6] A. Tahat, Y. Kheetan, and A. Sacca. Blood Pressure Measurement and Management Telemedicine System Based on a Smart-Phone. International Journal of Online and Biomedical Engineering, Vol. 9, no. 5, Sep. 2013. https://doi.org/10.3991/ijoe.v9i5.2697

[7] S. A. Hoeksel, J. A. Blom, J. R. Jansen, J. G. Maessen, and J. J. Schreuder. Automated infusion of vasoactive and inotropic drugs to control arterial and pulmonary pressures during cardiac surgery. Crit. Care Med. 27:2792-2798, 1999. https://doi.org/10.1097/00003246$\underline{199912000-00031}$

[8] R. R. Rao, B. W. Bequette, and R. J. Roy. Simultaneous regulation of hemodynamic and anesthetic states: A simulation study. Ann. Biomed. Eng. 28:71-84, 2000. https://doi.org/ $\underline{10.1114 / 1.255}$

[9] J. B. Slate, Model-based design of a controller for infusing nitroprusside during postsurgical hypertension. Ph.D. dissertation, University of Wisconsin-Madison, USA, 1980. 
[10] A. L. Maitelli, T. Yoneyama, Suboptimal Dual Adaptive Control for blood pressure management, IEEE Transactions on Biomedical Engineering, vol. 44, no. 6, pp. 486-492, Jun. 1997. https://doi.org/10.1109/10.581942

[11] H. A. Silva, Multi-Model Generalized Predictive Controller Applied to Blood Pressure Control (in Portuguese). Master's Thesis, Department of Electrical and Computer Engineering, Universidade Federal do Rio Grande do Norte, RN, Brazil, 2010. https://doi.org/ $\underline{10.24873 / \text { j.rpemd.2019.05.446 }}$

[12] H. A. Silva, C. P. Leão, E. A. Seabra, Parametric Sensitivity Analysis of a Multiple Model Adaptive Predictive Control for Regulation of Mean Arterial Blood Pressure. 15th International Conference on Informatics in Control, Automation and Robotics, ICINCO, vol. 1, pp. 510-516, Porto, Portugal, 2018. https://doi.org/10.5220/0006909805100516

[13] K. E. Barrett, S. M.Barman, S. Boitano, et al., Ganong's Review of Medical Physiology 26rd edition, MCGRAW-HILL EDUCATION - EUROPE, pp. 489-569, 2019.

[14] M. C.K. Khoo. Physiological Control Systems: Analysis, Simulation, and Estimation, 2nd Ed., IEEE Press Series in Biomedical Engineering, pp. 13-271, 2018.

[15] P. K. Whelton, R. M. Carey, W. S. Aronow, et al., Guideline for the Prevention, Detection, Evaluation, and Management of High Blood Pressure in Adults. A Report of the American College of Cardiology/American Heart Association Task Force on Clinical Practice Guidelines. Vol. 71, pp. 13-115, 2017. https://doi.org/10.22141/2307-1257.7.1.2018.122220

[16] O. J. M. Smith, Closed control of loops with dead-time. Chem. Eng. Process, vol. 53, pp. 217-219, 1957.

[17] J. E. Normey-Rico, E. F. Camacho, A Smith Predictor Based Generalized Predictive Controller. Technical Report GAR 1996/02, University of Seville, 1996.

[18] J. E. Normey-Rico, E. F. Camacho, J. G. Ortega, A Smith Prediction Based Generalized Predictive Controller for Mobile Robot Path Tracking. III IFAC Symposium on Intelligent Autonomous Vehicles, Madri, Spain, pp. 471-476, 1998. https://doi.org/10.1016/s1474$\underline{6670(17) 44077-8}$

[19] J. Zhu, G. Yang, Y. Zi and Y. Fang. Radio Networks Predictive Control of a T-type Inverter in Wind Energy. International Journal of Online and Biomedical Engineering, Vol. 12, no. 12, 2016.

[20] R. R. Rao, B. Aufderheide and B. W. Bequettey. Experimental Studies On Multiple \{Model Predictive Control For Automated Regulation of Hemodynamic Variables Ieee Trans. Biomed. Eng., Vol. 50 , no. 3, pp. 277-288, Mar. 2003. https://doi.org/10.1109 thme.2003.808813

[21] H. A. Silva, A. L. Maitelli, C. P. Leão, E. A. Seabra, Multiple Model SPGPC for Blood Pressure Control. 12th International Conference on Informatics in Control, Automation and Robotics, ICINCO, vol. 1, pp. 563-568, Colmar, France, 2015. https://doi.org/10.52 20/0005540805630568

[22] K.Y. Rani, H. Unbehauen, Study of predictive controller tuning methods. Automatica, vol. 33, no. 12, pp. 2243-2248, Dec. 1997. https://doi.org/10.1016/s0005-1098(97)00134-9

[23] W. G. He, H. Kaufman, R. Roy, Multiple Model Adaptive Control Procedure for Blood Pressure Control. IEEE Trans. on Biomed. Eng., vol. BME 33, no. 1, Jan. 1986. https://doi. org/10.1109/tbme. 1986.325833

[24] D. A. Linkens and S. S. Hacisalihzade, Computer control systems and pharmacological drug administration: a survey. Journal of Medicine Engineering Technology, vol. 14, no. 2, pp. 41 \{54, Jul. 1990. https://doi.org/10.3109/03091909009019266 


\section{Authors}

Humberto A. Silva (corresponding author) is Professor at the Federal Institute of Rio Grande do Norte, RN, Brazil. (e-mail: humberto.araujo@ifrn.edu.br)

Celina P. Leão Is Professor and Researcher in Algoritmi Centre, Engineering School of Minho University, Guimarães, Portugal. (e-mail: cpl@dps.uminho.pt)

Eurico A. Seabra Is Professor and Researcher in Metrics Center, Engineering School of Minho University, Guimarães, Portugal. (e-mail: eseabra@dem.uminho.pt)

Article submitted 2019-05-23. Resubmitted 2019-06-31. Final acceptance 2019-06-31. Final version published as submitted by the authors. 